\title{
Preliminary Studies on the Toxic Effects of Degradation Products of Oxytetracycline and Chlortetracycline on
}

\section{Rats}

\author{
Nguyen Van Hue ${ }^{1,2}$, Nguyen Van Toan ${ }^{2}$, Le Thanh Long ${ }^{2}$, Guido Fleischer ${ }^{1}$ and Zhou Guang Hong ${ }^{1}$ \\ 1. Key Laboratory of Meat Processing and Quality Control (MOE), National Center of Meat Quality and Safety Control (MOST), \\ College of Food Science and Technology, Nanjing Agricultural University, Nanjing 210095, China \\ 2. College of Agriculture and Forestry, Hue University, 102 Phung Hung Street, Hue City 47000, Vietnam
}

\begin{abstract}
The paper aimed to study the toxic effects of the degradation products of oxytetracycline and chlortetracycline, such as, $\alpha$-apo-oxytetracycline $\quad(\alpha$-apo-OTC), $\quad \beta$-apo-oxytetracycline $\quad(\beta$-apo-OTC), anhydro-chlortetracycline $\quad($ ACTC) and 4epi-anhydro-chlortetracycline (EACTC) on rats. Male rats received oral doses of $10 \mathrm{mg} / \mathrm{kg}$ body weight/day of either $\alpha$-apo-OTC, $\beta$-apo-OTC, ACTC or EACTC for $90 \mathrm{~d}$. At the end of $90 \mathrm{~d}$ treatment, the body/organ weight, white blood cell count (WBC), red blood cell count (RBC), hemoglobin (HGB), hematocrit (HCT), platelet (PLT) count, blood urea nitrogen (BUN), serum glutamic oxaloacetic transaminase (SGOT) and serum glutamic pyruvic transaminase (SGPT) were measured. The results revealed that the rats treated with $\alpha$-apo-OTC or ACTC for $90 \mathrm{~d}$ did not affect body, organ weights or certain blood- and serum-factors, however, those treated with $\beta$-apo-OTC or EACTC exhibited significant decreases in body weights, RBC counts and HGB concentrations, as well as significant increases in BUN concentrations, SGOT and SGPT activities. The results of this study suggested that the toxic effects of $\beta$-apo-OTC or EACTC treatment could damage liver and kidney tissues of rats, as well as lead to the degeneration and necrosis in the hepatocytes.
\end{abstract}

Key words: Antibiotic, oxytetracycline and chlortetracycline degradation products, toxic effects, rats.

\section{Introduction}

Oxytetracycline and chlortetracycline are broad-spectrum antibiotics and are members of the tetracycline family. Their therapeutic use has been of particular interest for food producing animals because of their broad-spectrum activity and low cost for preventing and controlling diseases, and also used as feed additives for the promotion of weight gain. However, their over and improper use can result in the presence of their residues in edible tissues of animals, which can be toxic and dangerous for human health and potentially cause allergic reactions. Liver plays a central role in detoxification and excretion of many endogenous and exogenous compounds. Hence, any injury to it or impairment of its function has grave

Corresponding author: Nguyen Van Hue, Ph.D., research field: food and chemical toxicology. implication for the health of the affected person. The mechanisms involved in liver injury are complex and interactive, and can be artificially separated as chemical and immune injuries. The biochemical mechanisms concern various chemicals that are detoxified in the liver via cytochrome P-450 and conjugation. Toxic metabolites may alter plasma membrane, mitochondria, intracellular ion homeostasis, or degratative enzyme activity. Immune mechanisms involve cell cooperation, and are mediated by cytokines, nitric oxide and complement. Pathologic apoptosis is potentially an important mechanism of acute liver injury (hypoxia/reoxygenation, liver congestion, acetaminophen poisoning, posttransplant acute liver rejection, severe sepsis, viral hepatitis and alcoholic liver disease) [1]. In an earlier report, the administration of therapeutic doses of 
tetracycline to male rats induced oxidative stress by decreasing the antioxidant system, increased lipid peroxidation and concomitantly impaired testicular function, spermatogenesis and epididymal sperm physiology and morphology [2]. In another report, tetracycline caused hyperglycemia and injury to the pancreas, and caused significant oxidative stress to liver and pancreas by reducing the levels of antioxidant enzymes and glutathione (GSH) and elevating the levels of lipid peroxide formed. In addition, this drug induced an hepatotoxicity caused by fatty infiltration of the liver and damage to liver parenchymal cells [3]. Usually, meat is cooked before consumption, and therefore oxytetracycline, chlortetracycline as well as any degradation products formed could be subjected to consumption, which may be a threat to human health. Tetracycline degradation products have been found in foods after treatment with different thermal processing conditions [4-6]. Degraded tetracycline preparations have been associated with Fanconi-type syndrome-a reversible renal dysfunction [7]. Benitz and Diermeier [8] reported that anhydro-4-epi-tetracycline was the only degradation product which caused abnormal urinary functions similar to the Fanconi-type syndrome. Severe nephrotic changes were found in the kidneys of rats after being given very large oral doses $(1,000$ $\mathrm{mg} / \mathrm{kg} / \mathrm{d}$ ), and in dogs after intravenous doses of 10 $\mathrm{mg} / \mathrm{kg}$ and $20 \mathrm{mg} / \mathrm{kg}$ on successive days. So far, few reports are available for toxicity of degradation products of tetracycline, chlortetracycline and oxytetracycline on sludge and soil bacteria [9], as well as phytoplanktons [10], still no reports are available in rats. Therefore, the objective of this study was to determine the toxicity of oxytetracycline and chlortetracycline degradation products when they were orally administered to rats.

\section{Materials and Methods}

\subsection{Chemicals}

The anhydro-chlortetracycline

(ACTC), 4-epianhydro-chlortetracycline

(EACTC), $\alpha$-apo-oxytetracycline $\quad(\alpha$-apo-OTC $) \quad$ and $\beta$-apo-oxytetracycline ( $\beta$-apo-OTC) were purchased from Acros Organics (Geel, Belgium).

\subsection{Animals}

A total of 50 healthy rats (4-week-old male Sprague-Dawley) were individually housed in stainless steel cages lined with wood shavings and fitted with wire mesh tops. The rats were acclimated to ambient temperature $\left(23 \pm 2{ }^{\circ} \mathrm{C}\right)$, humidity and natural light/dark cycle. All rats were fed a standard rat chow, had free access to drinking water and were kept in the facilities for at least one week prior to use. After confirming their normal health status at the end of the acclimation period, 50 rats were randomly allocated to five groups, each consisting of 10 rats. Then they were then given the control or experimental diets for $90 \mathrm{~d}$. The rats in group I served as control. The rats in groups II, III, IV and V received orally and daily $10 \mathrm{mg} / \mathrm{kg}$ body weight of $\alpha$-apo-OTC, $\beta$-apo-OTC, ACTC or EACTC, respectively.

\subsection{Clinical Observations}

The behavior and appearance of all rats, including coat condition, skin, eyes and excretions, were monitored every $2 \mathrm{~d}$ throughout the study. Body, organ weights and macroscopic observation of liver were conducted on all surviving rats on the 90th day (the end of the trial).

\subsection{Biochemical Analysis}

Hematology and serum chemistry were conducted on all surviving rats on the 90th day (the end of the trial). Rats were fasted for $12 \mathrm{~h}$ before collecting blood samples. Whole blood from the inner canthus was collected with and without anticoagulant, and analyzed for white blood cell count (WBC), red blood cell count (RBC), hemoglobin (HGB), hematocrit (HCT), platelet count (PLT), urea nitrogen (BUN), serum glutamic oxaloacetic transaminase (SGOT) and 
serum glutamic pyruvic transaminase (SGPT).

\subsection{Statistical Analysis}

The data were present as mean value and standard deviation (SD). A one-way analysis of variance (ANOVA) was applied to evaluate the homogeneity variance of all of groups. ANOVA was used to compare the experimental groups. The response variable values of the treatment groups were compared to the control group using $t$-test. Differences between values were considered statistically significant at $P<0.05$. All analysis were carried out using the SPSS statistics software version 20.

\section{Results and Discussion}

\subsection{Body and Organ Weights}

The body weights, organ weights and their percentages are presented in Table 1. No significant differences in body weight were observed between control rats and treated rats with $\alpha$-apo-OTC or ACTC. In contrast, the body weights of those rats treated with $\beta$-apo-OTC or EACTC showed significant changes compared with rats in the control group $(P<0.05)$. The groups treated with $\beta$-apo-OTC or EACTC showed significant increase in both absolute and relative weights of the liver and kidney as compared with the control group, whereas, no significant changes were observed in the weights of liver and kidney in the group treated with $\alpha$-apo-OTC or ACTC, as compared with control group (Table 1).

Absolute heart weight was significantly decreased, whereas, the significantly increased changes were observed in the relative weight of lung in the group treated with $\beta$-apo-OTC, as compared with control group. Absolute and relative heart/testis weights in the groups treated with $\alpha$-apo-OTC or ACTC were slightly decreased, but no significant difference was detected compared with the control group.

\subsection{Hematology}

Hematological analysis from the groups treated with $\beta$-apo-OTC or EACTC were significantly different from those obtained from the control group (Table 2). However, no differences were observed between rats in the control group and those in the groups treated with $\alpha$-apo-OTC or ACTC. In the groups treated with $\beta$-apo-OTC or EACTC, there was significant increase $(P<0.001)$ in both WBC and PLT, as compared with the control animals. Although the WBC and PLT of rats in the groups treated with $\alpha$-apo-OTC or ACTC were also slightly increased, no significant differences were detected compared with controls. RCB, HGB and HCT in the groups treated with $\beta$-apo-OTC, EACTC were significantly decreased

Table 1 Body weight, organs weight and percentage of organs weight per body weight in male rats treated with antibiotics as indicated in mean $\pm \operatorname{SD}(n=10)$.

\begin{tabular}{llllll}
\hline Parameter & Control & $\alpha$-apo-OTC & $\beta$-apo-OTC & ACTC & EACTC \\
\hline Body weight (g) & $359.02 \pm 18.98$ & $354.33 \pm 20.26$ & $341.57 \pm 14.34^{*}$ & $348.92 \pm 21.27$ & $338.97 \pm 20.36^{*}$ \\
Liver weight (g) & $13.43 \pm 1.59$ & $14.16 \pm 1.84$ & $14.97 \pm 1.09^{*}$ & $14.34 \pm 1.06$ & $15.84 \pm 2.23^{*}$ \\
Liver weight (\%) & $3.76 \pm 0.58$ & $4.02 \pm 0.61$ & $4.39 \pm 0.39^{*}$ & $4.12 \pm 0.39$ & $4.71 \pm 0.82^{* *}$ \\
Kidney weight (g) & $1.86 \pm 0.14$ & $1.93 \pm 0.15$ & $2.08 \pm 0.15^{* *}$ & $2.00 \pm 0.17$ & $2.13 \pm 0.11^{* * *}$ \\
Kidney weight (\%) & $0.52 \pm 0.03$ & $0.55 \pm 0.06$ & $0.61 \pm 0.05^{* * *}$ & $0.57 \pm 0.04$ & $0.63 \pm 0.04^{* * *}$ \\
Lungs weight (g) & $1.32 \pm 0.12$ & $1.35 \pm 0.07$ & $1.34 \pm 0.10$ & $1.39 \pm 0.09$ & $1.36 \pm 0.12$ \\
Lungs weight (\%) & $0.37 \pm 0.04$ & $0.38 \pm 0.04$ & $0.39 \pm 0.03^{*}$ & $0.40 \pm 0.04$ & $0.40 \pm 0.04$ \\
Heart weight (g) & $0.96 \pm 0.08$ & $0.92 \pm 0.11$ & $0.88 \pm 0.09^{*}$ & $0.88 \pm 0.10$ & $0.87 \pm 0.12$ \\
Heart weight (\%) & $0.27 \pm 0.03$ & $0.26 \pm 0.03$ & $0.26 \pm 0.03$ & $0.25 \pm 0.03$ & $0.26 \pm 0.03$ \\
Testis weight (g) & $2.32 \pm 0.18$ & $2.29 \pm 0.15$ & $2.16 \pm 0.18$ & $2.21 \pm 0.18$ & $2.11 \pm 0.18^{*}$ \\
Testis weight (\%) & $0.65 \pm 0.06$ & $0.65 \pm 0.05$ & $0.63 \pm 0.04$ & $0.64 \pm 0.06$ & $0.62 \pm 0.08$ \\
\hline Rats were
\end{tabular}

Rats were treated orally and daily for $90 \mathrm{~d}$ by $10 \mathrm{mg} / \mathrm{kg}$ body weight of $\alpha$-apo-OTC, $\beta$-apo-OTC, ACTC or EACTC.

$*, * *$ and $* * *$ mean significant difference at $P<0.05, P<0.01$ and $P<0.001$ level, respectively, when compared with the control group. 
Table 2 Hematological analyses of male rats after $90 \mathrm{~d}$ feeding (mean \pm SD) $(n=10)$.

\begin{tabular}{llllll}
\hline Parameter & Control & $\alpha$-apo-OTC & $\beta$-apo-OTC & ACTC & EACTC \\
\hline WBC $\left(\times 10^{9} / \mathrm{L}\right)$ & $5.08 \pm 0.29$ & $5.50 \pm 0.74$ & $6.46 \pm 0.67^{* * *}$ & $5.21 \pm 0.60$ & $6.71 \pm 0.91^{* * *}$ \\
RBC $\left(10^{12} / \mathrm{L}\right)$ & $8.03 \pm 0.76$ & $8.43 \pm 0.50$ & $6.93 \pm 0.65^{* *}$ & $7.87 \pm 0.70$ & $6.30 \pm 0.60^{* * *}$ \\
HGB $(\mathrm{g} / \mathrm{dL})$ & $13.61 \pm 1.11$ & $12.61 \pm 1.27$ & $10.96 \pm 1.12^{* * *}$ & $13.62 \pm 1.22$ & $11.22 \pm 1.46^{* *}$ \\
HCT $(\%)$ & $48.54 \pm 6.74$ & $48.75 \pm 3.19$ & $41.68 \pm 3.52^{*}$ & $44.10 \pm 3.40$ & $40.08 \pm 4.38^{* *}$ \\
PLT $\left(\times 10^{9} / \mathrm{L}\right)$ & $451.31 \pm 47.60$ & $489.41 \pm 50.56$ & $542.28 \pm 41.60^{* * *}$ & $479.63 \pm 45.87$ & $586.84 \pm 37.97^{* * *}$ \\
\hline
\end{tabular}

Rats were treated orally and daily by $10 \mathrm{mg} / \mathrm{kg}$ body weight of $\alpha$-apo-OTC, $\beta$-apo-OTC, ACTC or EACTC.

WBC: white blood cell count; RBC: red blood cell count; HGB: hemoglobin; HCT: hematocrit; PLT: platelet count.

*** and ${ }^{* *}$ mean significant difference at $P<0.05, P<0.01$ and $P<0.001$ level, respectively, when compared with the control group.

Table 3 Serum enzyme activities and urea nitrogen in male rats after $90 \mathrm{~d}$ of feeding $(\mathrm{mean} \pm \mathrm{SD})(\mathrm{n}=10)$.

\begin{tabular}{llllll}
\hline Parameter & Control & $\alpha$-apo-OTC & $\beta$-apo-OTC & ACTC & EACTC \\
\hline SGOT (U/L) & $50.22 \pm 4.25$ & $54.01 \pm 3.84$ & $74.38 \pm 13.37^{* * *}$ & $52.15 \pm 5.72$ & $65.12 \pm 10.07^{* * *}$ \\
SGPT (U/L) & $43.29 \pm 6.12$ & $45.02 \pm 5.33$ & $58.82 \pm 9.48^{* * *}$ & $46.54 \pm 6.29$ & $61.78 \pm 5.85^{* * *}$ \\
BUN (mg/dL) & $51.54 \pm 8.81$ & $46.86 \pm 4.09$ & $64.24 \pm 9.49^{* *}$ & $55.00 \pm 5.65$ & $69.84 \pm 8.50^{* * *}$ \\
\hline
\end{tabular}

Rats were treated orally and daily by $10 \mathrm{mg} / \mathrm{kg}$ body weight of $\alpha$-apo-OTC, $\beta$-apo-OTC, ACTC or EACTC.

BUN: urea nitrogen; SGOT: serum glutamic oxaloacetic transaminase; SGPT: serum glutamic pyruvic transaminase.

$*, * *$ and $* * *$ mean significant difference at $P<0.05, P<0.01$ and $P<0.001$ level, respectively, when compared with the control group.

when compared with that of the control group, whereas no significant changes occurred in the rats treated with $\beta$-apo-OTC or EACTC compared with control group.

\subsection{Serum Chemistry and Macroscopic Changes}

The effects of the tetracycline degradation products on various serum constituents are given in Table 3. Significant differences in some constituents were observed between rats in the control group and those in the groups treated with $\beta$-apo-OTC or EACTC. However, changes in the serum constituents of the groups treated with $\alpha$-apo-OTC or ACTC did not show any significant differences compared with the controls. Both SGOT and SGPT in the groups treated with $\beta$-apo-OTC or EACTC were significantly increased in compared with the controls $(P<0.001)$. Although the SGOT and SGPT of rats in the groups treated with $\alpha$-apo-OTC or ACTC were also slightly increased, but not significant compared with controls. The administration of $\beta$-apo-OTC significantly increased BUN concentration $(P<0.01)$ compared to the control group, whereas the BUN in the group treated with EACTC was significantly increased $(P<$
0.001) in comparison with that of the control group.

At the 90th day, degeneration and necrosis were observed in the hepatocytes of rats in the groups treated with either $\beta$-apo-OTC or EACTC (Fig. 1). While no necrosis was observed in the livers of rats in the groups treated with $\alpha$-apo-OTC or ACTC, other morphological changes occurred.

The present study indicated that when a dose of either $\beta$-apo-OTC or EACTC (10 mg/kg body weight) orally administered for $90 \mathrm{~d}$, the body weight of rats significantly decreased, and concomitantly there were significant increases in both the absolute and relative weights of the liver and kidney as compared with the control. This did not result from malnutrition, since they had a normal daily food intake. These findings demonstrate that both $\beta$-apo-OTC and EACTC have toxic effects of on rats. Further, both the $\beta$-apo-OTC and EACTC treated rats showed a decrease in the RBC count and HGB concentration in their blood, whereas the WBC count and the PLT count were both significantly increased compared with the control group.

In the present study, serum SGOT, SGPT and BUN activities were used as markers of liver and kidney 

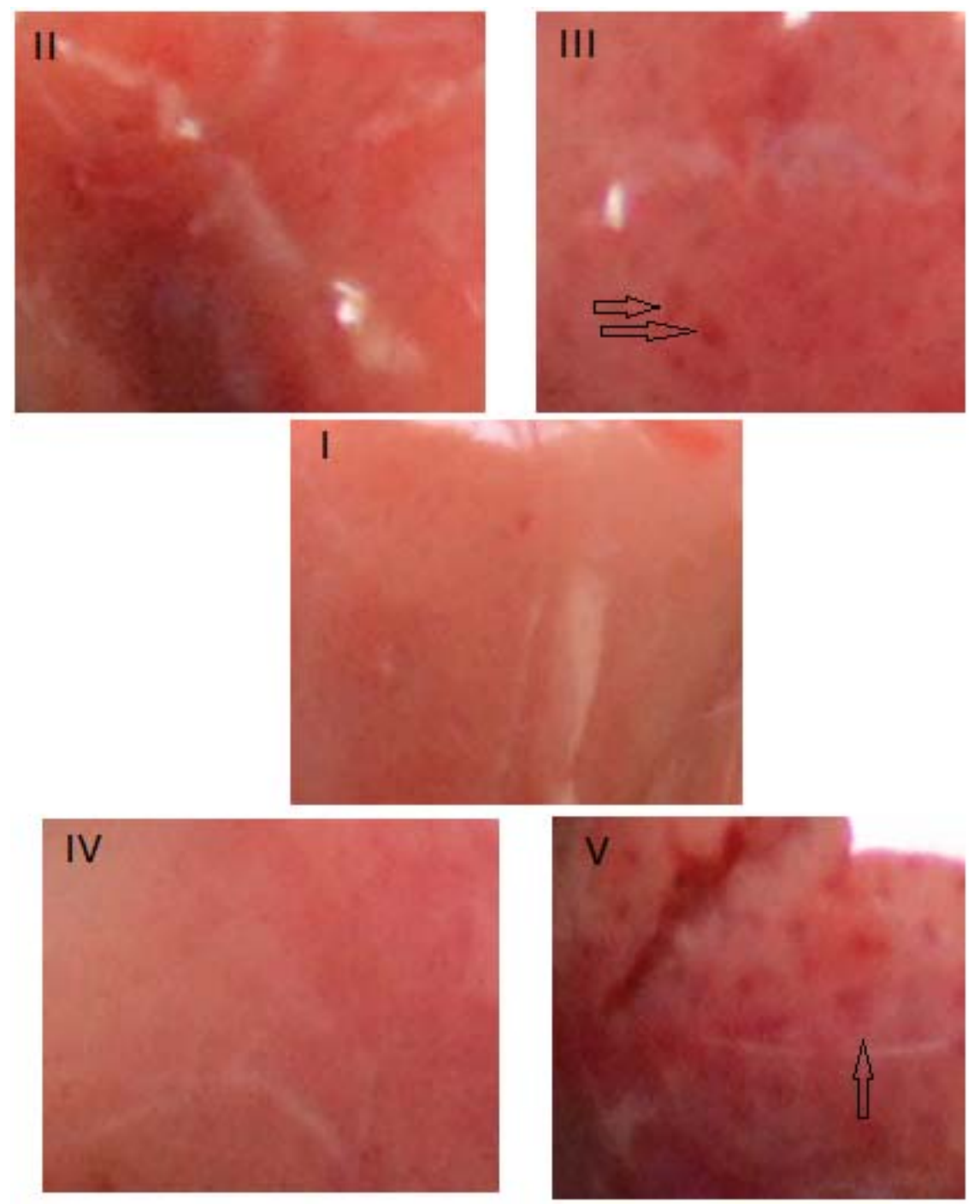

Fig. 1 Representative images of livers from rats.

I: control rat showing normal morphology; II: rats treated with $\alpha$-apo-OTC; III: rat treated with $\beta$-apo-OTC; IV: rat treated with ACTC; V: rat treated with EACTC.

damage. The animals treated with either $\beta$-apo-OTC or EACTC showed a significant increased in serum SGOT, SGPT and BUN. These data demonstrate that both $\beta$-apo-OTC and EACTC were toxic and appear to damage liver and kidney rats. The tetracycline degradation product, anhydro-4-epi-tetracycline was the only product which caused abnormal urinary findings of a type similar to the Fanconi-type syndrome. Severe nephrotic changes were found in the kidneys of rats and dogs treated with anhydro-4-epi-tetracycline [7]. Although several of the degradation products, including anhydrotetracycline
(ATC) and ACTC, have been shown to be potent antibiotics on tetracycline resistant bacterial strains, and on the other hand, the products of chlortetracycline have adverse effects on fresh-water phytoplankton [8,9], yet no studies have been conducted on the toxicity of the degradation products of oxytetracycline and chlortetracycline in animals.

\section{Conclusions}

The present study was designed to determine the toxicity of oxytetracycline and chlortetracycline degradation products upon oral administration to rats. 
The results demonstrated that the rats treated with $\alpha$-apo-OTC or ACTC for $90 \mathrm{~d}$ did not affect body, organ weights or certain blood- and serum-factors. The $\beta$-apo-OTC and EACTC, on the other hand, exhibited significantly toxic effects on male rats, significantly decreased body weights and RBC counts, lowered HGB concentrations and increased WBC and PLT counts. Concomitantly, there were increases in serum SGOT, SGPT and BUN. In summary, the toxic effects of $\beta$-apo-OTC and EACTC in rats appeared to damage the liver and kidney, which ultimately led to necrosis of the hepatocytes. Further studies are suggested to explore the mechanisms of liver and kidney toxicity by $\beta$-apo-OTC and EACTC.

\section{References}

[1] Losser, M. R., and Payen, D. 1996. "Mechanisms of Liver Damage.” Semin. Liver Dis. 16 (4): 357-67.

[2] Farombi, E. O., Ugwuezunmba, M. C., Ezenwadu, T. T., Oyeyemi, M. O., and Ekor, M. 2008. "Tetracycline-Induced Reproductive Toxicity in Male Rats: Effects of Vitamin C and N-Acetylcysteine." Experimental and Toxicologic Pathology 60 (1): 77-85.

[3] Asha, K. K., Sankar, T. V., and Nair, P. G. 2007. "Effect of Tetracycline on Pancreas and Liver Function of Adult Male Albino Rats.” J. Pharm Pharmacol. 59 (9): 1241-8.

[4] Kuhne, M., Hamscher, G., Korner, U., Schedl, D., and
Wenzel, S. 2001. "Formation of Anhydrotetracycline during a High-Temperature Treatment of Animal-Derived Feed Contaminated with Tetracycline." Food Chem. 75 (4): 423-9.

[5] Gratacós-Cubarsí, M., Fernandez-Garcia, A., Picouet, P., Valero-Pamplona, A., Garcia-Regueiro, J. A., and Castellari, M. 2007. "Formation of Tetracycline Degradation Products in Chicken and Pig Meat under Different Thermal Processing Conditions.” J. Agric. Food Chem. 55 (11): 4610-6.

[6] Fedeniuk, R. W., Shand, P. J., and McCurdy, A. R. 1997. "Effect of Thermal Processing and Additives on the Kinetics of Oxytetracycline Degradation in Pork Muscle." J. Agric. Food Chem. 45 (6): 2252-7.

[7] Frimpter, G. W., Timpanelli, A. E., Eisenmenger, W. J., Stein, H. S., and Ehrlich, L. I. 1963. "Reversible 'Fanconi Syndrome' Caused by Degraded Tetracycline.” JAMA. 184 (2): 111-3.

[8] Benitz, K. F., and Diermeier, H. F. 1964. "Renal Toxicity of Tetracycline Degradation Products." Proc. Soc. Exp. Biol. Med. 115 (4): 930-5.

[9] Halling-Sorensen, B., Sengelov, G., and Tjornelund, J. 2002. "Toxicity of Tetracyclines and Tetracycline Degradation Products to Environmentally Relevant Bacteria, Including Selected Tetracycline-Resistant Bacteria." Arch. Environ. Contam. Toxicol. 42 (3): 263-71.

[10] Guo, R. X., and Chen, J. Q. 2012. "Phytoplankton Toxicity of the Antibiotic Chlortetracycline and Its UV Light Degradation Products." Chemosphere 87 (11): 1254-9. 